TIP Periodica Polytechnica

Transportation Engineering

43(1), pp. 27-34, 2015

DOI: 10.3311/PPtr.7542

Creative Commons Attribution (i)

RESEARCH ARTICLE

\section{Symmetric Stiffened Shell Structures}

\author{
Péter HARTH ${ }^{1, *}$, Pál MICHELBERGER $\dagger^{2}$
}

Received 30 May 2014

\begin{abstract}
In this paper the analysis of the symmetric stiffened shell structure are introduced. The main purpose of this paper is to reduce the number of the unknowns in the compatibility equation to ease vehicle preliminary design process. One shell field contains a square-shaped shell and four rod with joints in the corner. Different cases are examined depending on the number of the shells.
\end{abstract}

\section{Keywords}

symmetric stiffened shell structure, vehicle preliminary design, indetermined shell structures

\section{Nomenclatures}

a Side size of the shell $[\mathrm{m}]$

$\mathrm{v}$ thickness of the shell $[\mathrm{m}]$

A Area of the shell $\left[\mathrm{m}^{2}\right]$

E Young Modulus [MPa]

G Shear Modulus [MPa]

i Element of the set I [-]

$\mathrm{j} \quad$ Element of the set $\mathrm{J}[-]$

$\mathrm{k} \quad$ Element of the set $\mathrm{K}[-]$

$\mathrm{m} \quad$ Number of the rows [-]

n Number of the columns [-]

I Set for the unknown ident. [-]

J Set for the unknown ident. [-]

K Set for the unknown ident. [-]

$\mathrm{M}_{\mathrm{SS}}$ Comp. matrix of the SS groups [Nm]

$\mathrm{M}_{\mathrm{SA}}$ Comp. matrix of the SA groups [Nm]

$\mathrm{M}_{\mathrm{AS}}$ Comp. matrix of the AS groups [Nm]

$\mathrm{M}_{\mathrm{AA}}$ Comp. matrix of the AA groups [Nm]

$\mathrm{M}_{4 \mathrm{~S}}$ Comp. matrix of the $4 \mathrm{~S}$ groups [Nm]

$\mathrm{M}_{2 \mathrm{~S} 2 \mathrm{~A}}$ Comp. matrix of the 2S2A groups [Nm]

$\mathrm{M}_{2 \mathrm{~A} 2 \mathrm{~S}}$ Comp. matrix of the $2 \mathrm{~A} 2 \mathrm{~S}$ groups [Nm]

$\mathrm{M}_{4 \mathrm{~A}}$ Comp. matrix of the 4A groups $[\mathrm{Nm}]$

$\delta p q$ Coeff. of the comp. matrix $(p=q)[N m]$

$\delta p q \quad$ Coeff. of the comp. matrix $(p \neq q)[N m]$

\section{Introduction}

The stiffened shell structures appear quite a lot place in engineering practice. These can be applied in various shapes in road and railway vehicle design as well. The generally used shapes are rectangular and square-shaped. Lucas dealt road and rail vehicles by analyzing the Argyris method (Lucas 1959; 1960; 1963).

In this paper the square-shaped shell structures examination is introduced, supported by symmetry planes. Adeleke investigated some symmetry transformation of shells. This transformation can be used in a semi-invers method for studying materials. (Adeleke 1983; 1985). Thin-walled weakly conical and cylindrical shell with arbitrary open simply or multiply closed contour of transverse cross-section strengthened by longitudinal elements was examined by (Tyutyunnikov, Shklyarchuk, 2008). 
Local symmetry groups of dynamically and kinematically exact theory of elastic shells have been established. Definitions of the fluid, the solid, and the membrane shell was introduced in (Eremeyev, Pietraszkiewicz, 2006).

In our examination shells are planar structures and stiffened with rods, joined by perfect frictionless joints each other. The examined shell structures are built of $m \times n$ shell fields. The number of the shell in column is denoted by $m$ and, in row is denoted by $n$. With this consideration there are three cases can be pass.

The first case is when $m=n$. These can be even or odd. If the number of the shells is even, symmetry planes are located between two shells, if odd the planes section the shells.

Let examine the following shell structure (Fig. 1). This structure contains 4 shells and 12 rods with joints. The rods and the shells contact only by shear. Two neighbor shells have a common rod. Furthermore, structure has 4 symmetry planes.

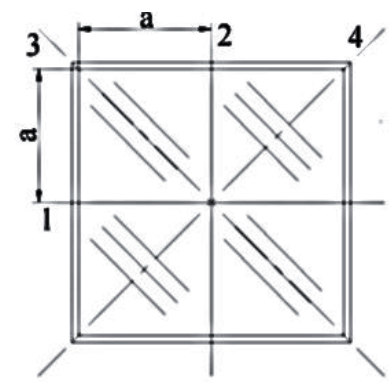

Fig. 1 Shell unit with 4 symmetry planes

If $m \neq n$, the shell structure has only 2 symmetry planes (Fig 2).

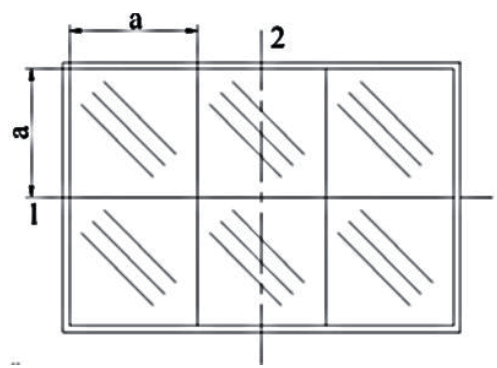

Fig. 2 Shell structure with 2 symmetry planes

\section{Structure of shell}

In our examination a $2 \times 2$ shell structure is considered like unit, called shell unit. This unit contains only one unknown which determines the normal load in rods and shear load in the shells. The number of the symmetry planes is 4 in shell unit. The inner load must be symmetric for all the symmetry planes ("1", "2", "3" and "4"). There are two type of shell unit; called symmetric and antimetric shell unit (Fig. 3 and Fig. 4). Every 4 neighbor shell creates an unknown.

The outer load can be antimetric also for the symmetry planes when $m=n>2$. The four symmetry states can be SSSS, SSAA, AASS and AAAA, where S means symmetry and A means antimetry plane. For example, the state of the symmetry is AASS that means the outer load is antimetric for the " 1 " and " 2 " planes and symmetric for the " 3 " and " 4 ".

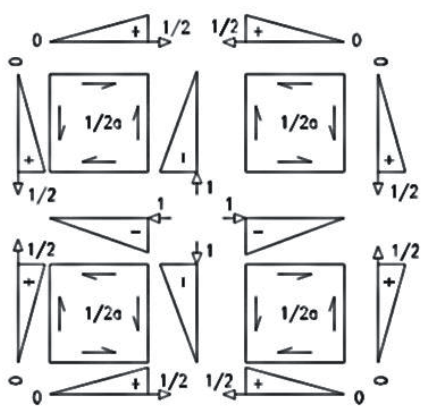

Fig. 3 Inner load in symmetric shell unit

Inner load can be described in other way; it is the antimetric pair of the symmetric shell unit (Fig. 4).
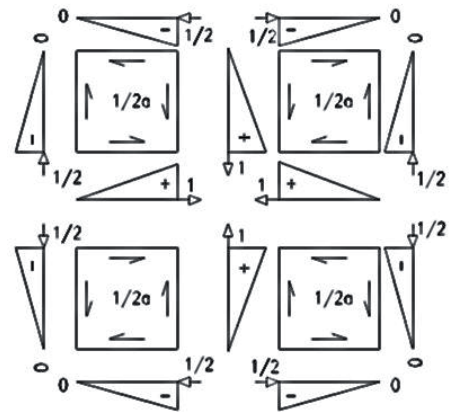

Fig. 4 Inner load in antimetric shell unit

The second case is when $m \neq n$ and one of $m$ or $n$ is 2 . Here the number of the symmetry planes is only 2 , because there is no opportunity to use the diagonal symmetry planes (" 3 " and "4"). The load can be symmetric and antimetric for all symmetry planes except that symmetry plane which is located between two shells. In other words, if a plane intersects an unknown, that plane must be symmetry plane. If not, the joints along the plane are not in static balance.

If $m \neq n$ and $m \neq 2$ and $n \neq 2$ then the load can be antimetric for the symmetry planes contrary the previous case. (In the following that can be seen, some unknowns do not meet the symmetry requirements).

The disassembled structures with rods and shells can be seen on Fig. 5 and Fig. 6. On the figure below can be seen if the load symmetric for " 1 "and " 2 ". The shear load is zero in the $(n+1) / 2$ th shells.

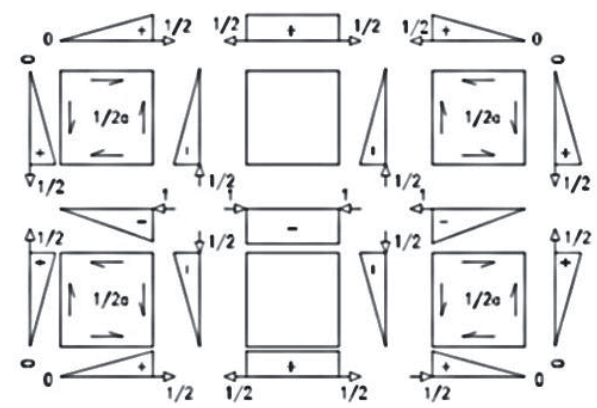

Fig. 5 Inner load in a 2x3 shell structure (SS--) 
In antimetric case shear load is double in the $(n+1) / 2$ th shells (Fig. 6).

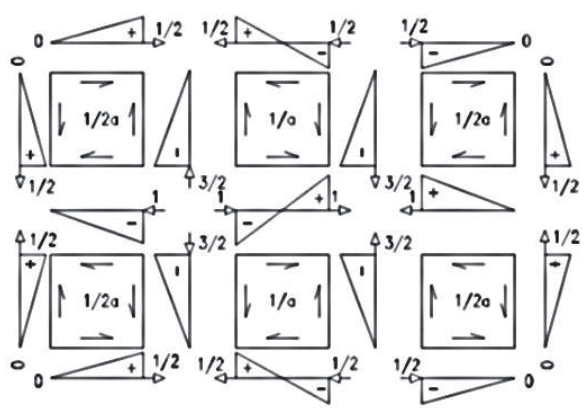

Fig. 6 Inner load in a $2 \times 3$ shell structure (SA--)

Examine the following 2x4 shell structure (Fig. 7). Previously, between two shells the load would have had to be symmetric, but here the load can be antimetric for " 2 ".

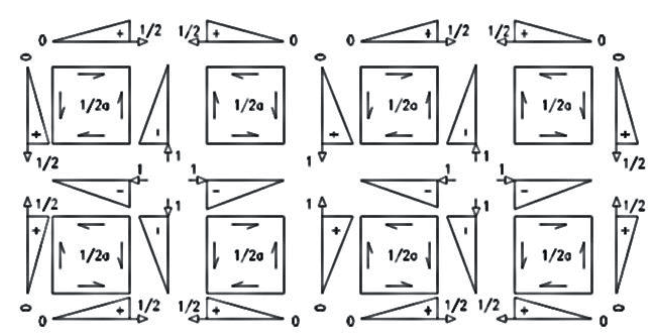

Fig. 7 Inner load in a $2 \times 4$ shell structure (SS--)

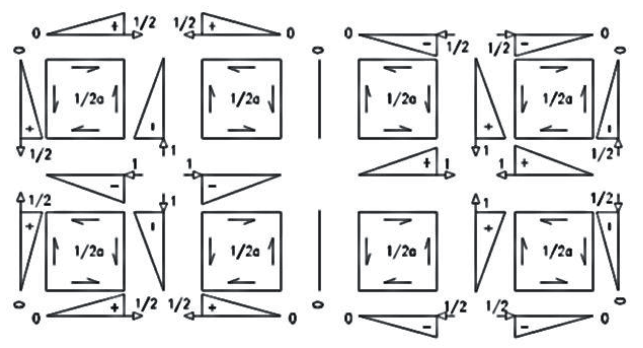

Fig. 8 Inner load in a 2x4 shell structure (SA--)

The $2 \times 4$ shell structure is built of two different $(2+1)$ shell units (Fig 7 and Fig. 8).

\section{Applicability of the symmetry planes}

Applicability of the symmetry planes are depending on the number of the shells. This declares which plane can be used during calculation. In the mentioned unit shells there are no antimetry, only symmetry planes. In other words, if $m=n=2$, between two shells the load can be only symmetric. If $m, n>2$ there is opportunity to apply symmetry and antimetry plane.

The table shows the applicability of the symmetry planes depending on number of the shells (Table 1).

Twofold symmetric $m \times n$ shell structure contains $(m-1) \times(n-1)$ unknowns. One shell field can be contained up to 4 unknowns. The locations of the unknowns ordering are from the first shell
Table 1 Applicability of the symmetry planes

\begin{tabular}{ccccc}
\hline $\boldsymbol{m} \boldsymbol{x} \boldsymbol{n}$ & "1" & "2" & "3" & "4" \\
\hline $2 \times 2$ & $\mathrm{~S}$ & $\mathrm{~S}$ & $\mathrm{~S}$ & $\mathrm{~S}$ \\
\hline $2 \times 3$ & $\mathrm{~S}$ & $\mathrm{~S} / \mathrm{A}$ & - & - \\
\hline $2 \times 4$ & $\mathrm{~S}$ & $\mathrm{~S} / \mathrm{A}$ & - & - \\
\hline $3 \times 3$ & $\mathrm{~S} / \mathrm{A}$ & $\mathrm{S} / \mathrm{A}$ & $\mathrm{S} / \mathrm{A}$ & $\mathrm{S} / \mathrm{A}$ \\
\hline $3 \times 4$ & $\mathrm{~S} / \mathrm{A}$ & $\mathrm{S} / \mathrm{A}$ & - & - \\
\hline $4 \times 4$ & $\mathrm{~S} / \mathrm{A}$ & $\mathrm{S} / \mathrm{A}$ & $\mathrm{S} / \mathrm{A}$ & $\mathrm{S} / \mathrm{A}$ \\
\hline $4 \times 5$ & $\mathrm{~S} / \mathrm{A}$ & $\mathrm{S} / \mathrm{A}$ & - & - \\
\hline $5 \times 5$ & $\mathrm{~S} / \mathrm{A}$ & $\mathrm{S} / \mathrm{A}$ & $\mathrm{S} / \mathrm{A}$ & $\mathrm{S} / \mathrm{A}$ \\
\hline$\cdot$ & $\cdot$ &. & $\cdot$ & $\cdot$ \\
\hline$m \neq n$ & $\mathrm{~S} / \mathrm{A}$ & $\mathrm{S} / \mathrm{A}$ & - & - \\
\hline$m=n$ & $\mathrm{~S} / \mathrm{A}$ & $\mathrm{S} / \mathrm{A}$ & $\mathrm{S} / \mathrm{A}$ & $\mathrm{S} / \mathrm{A}$ \\
\hline
\end{tabular}

in the first row. In the first row there are $n-1$ unknowns. Continuing the process in the last shell contains the $(m-1) \times(n-1)$ th unknown in the last row. These are examined in only one section, because all the unknowns are mapped from here.

In case of $m=n$ and higher order symmetry (SSSS, SSAA...), there is opportunity to complete with lower order symmetry.

During the preliminary design process, unknowns are divided into orthogonally groups to ease the calculation. The location of the symmetry and antimetry plane ("1", "2", "3" and"4") determines the requirements of the group. If one unknown meets this requirement, this group contains the mentioned unknown. All the unknowns can be divided only one orthogonally group.

Let see some examples. Asymmetric 3x3 shell structure contains 9 shells and 4 unknowns. Since $m$ and $n>2$ the planes can be both symmetry and antimetry. Thus all the symmetry states can be passed. There are three different orth. systems which are applicable in the same time. The first is when the " 1 " and "2" planes are applied, the second is when " 3 " and " 4 ", finally the third is when all planes are applied. In order the orthogonally groups are: SS--, SA--, AS--, AA--, --SS, --SA, --AS, --AA, SSSS, SSAA, AASS and AAAA.

It is necessary to make a decision which orthogonally system is practical during design process. If " 1 " and " 2 " symmetry planes are used, all the four orth. groups contain one unknown, this means four pieces one-unknowns equation. If " 3 " and "4" symmetry planes are used,the --SS orth. group contains 2 unknowns and there are two groups with one unknown, but --AA orth. groups does not exist. If all the symmetry planes are used ("1", "2", "3" and "4"), only two orth. groups are exist with one-one unknown (SSSS and AASS), but here must to complete with a lower order orth. groups.

Applying higher order symmetry not the all unknowns can be divided into orth. groups, thus there are some unknowns which do not meet symmetry requirements and in these cases is needed to complete with lower order symmetry. 
Table 2 The number of the unknowns in different orth. groups and shell numbers

\begin{tabular}{|c|c|c|c|c|c|c|c|c|c|c|c|c|c|c|}
\hline \multirow[b]{2}{*}{$\stackrel{\equiv}{\geqq}$} & \multirow[b]{2}{*}{ 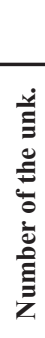 } & & \multicolumn{12}{|c|}{ Orth. systems and orth. groups } \\
\hline & & $\begin{array}{l}2 \\
3 \\
4\end{array}$ & - & $\begin{array}{l}\text { S } \\
\mathbf{A} \\
- \\
-\end{array}$ & $\begin{array}{l}\mathbf{A} \\
\mathbf{S} \\
- \\
-\end{array}$ & $\begin{array}{l}\mathbf{A} \\
\mathbf{A} \\
-\end{array}$ & $\begin{array}{l}- \\
- \\
\text { S } \\
\text { S }\end{array}$ & - & - & - & $\begin{array}{l}\mathrm{S} \\
\mathrm{S} \\
\mathrm{S} \\
\mathrm{S}\end{array}$ & $\begin{array}{l}\text { S } \\
\text { S } \\
\text { A } \\
\text { A }\end{array}$ & S & $\begin{array}{l}\mathbf{A} \\
\mathbf{A} \\
\mathbf{A} \\
\mathbf{A}\end{array}$ \\
\hline ઼ָ & 1 & & 1 & 0 & 0 & 0 & 1 & 0 & 0 & 0 & 1 & 0 & 0 & 0 \\
\hline$\underset{\sim}{\tilde{u}}$ & 2 & & 1 & 1 & 0 & 0 & & & & & & & & \\
\hline 艾 & 3 & & 2 & 1 & 0 & 0 & & & & & & & & \\
\hline$\stackrel{\substack{n \\
m}}{n}$ & 4 & & 1 & $\begin{array}{l}1 \\
\text { (1) }\end{array}$ & $\begin{array}{l}1 \\
\text { (1) }\end{array}$ & 1 & 2 & 1 & 1 & 0 & 1 & 0 & 1 & 0 \\
\hline$\underset{m}{~}$ & 6 & & 2 & 1 & 2 & 1 & & & & & & & & \\
\hline 齐 & 9 & & 4 & $\begin{array}{l}2 \\
\text { (2) }\end{array}$ & $\begin{array}{l}2 \\
\text { (2) }\end{array}$ & 1 & 4 & 2 & 2 & 1 & 3 & 1 & 1 & 0 \\
\hline$\stackrel{x}{x}$ & 12 & & 4 & 4 & 2 & 2 & & & & & & & & \\
\hline "n & 16 & & 4 & $\begin{array}{l}4 \\
\text { (4) }\end{array}$ & $\begin{array}{r}4 \\
\text { (4) }\end{array}$ & 4 & 6 & 4 & 4 & 2 & 3 & 1 & 3 & 1 \\
\hline 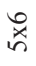 & 20 & & 6 & 4 & 6 & 4 & & & & & & & & \\
\hline גै & 25 & & 9 & $\begin{array}{l}6 \\
\text { (6) }\end{array}$ & $\begin{array}{c}6 \\
\text { (6) }\end{array}$ & 4 & 9 & 6 & 6 & 4 & 6 & 3 & 3 & 1 \\
\hline
\end{tabular}

The fourfold symmetric structure is indetermined at:

$$
\frac{m(m-2)}{2}+0,5
$$

if $m$ is odd and

$$
\frac{m(m-2)}{2}+1
$$

if $m$ is even.

The lower order groups cannot be optional; these must be SA-- and AS--. These lower order orth. groups cannot be passed in the higher one.

Back to our $3 \times 3$ example in the process of the preliminary design there is no different between the lower and higher order symmetry groups, because all can be solved with four pieces one-unknown equation system. The complementary unknowns are denoted by "(x)" in Table 2. In cases of $4 \times 4$ and lower order groups there are four-, two- and one-unknowns equation systems. Applied higher order, there are three-, two- and oneunknown equation system achieved easier computational work.
The number of the unknowns in different orth. groups can be calculated in general form (Table 3, 4, 5, and 6).

If " 1 " and " 2 " symmetry planes are used, number of the unknowns in the orth. groups:

Table 3 Number of the unknowns when $m=n$ or $m \neq n$ but both even or odd (Only "1" and "2" are used)

\begin{tabular}{lcccc}
\hline \multirow{\Sigma}{\Sigma}{} & SS- & SA-- & AS-- & AA- \\
\hline e. & $\frac{\boldsymbol{m}}{4}$ & $\frac{(n-2) m}{4}$ & $\frac{(m-2) n}{4}$ & $\frac{(m-2)(n-2)}{4}$ \\
\hline o. & $\frac{(m-1)(n-1)}{4}$ & $\frac{(m-1)(n-1)}{4}$ & $\frac{(m-1)(n-1)}{4}$ & $\frac{(m-1)(n-1)}{4}$ \\
\hline
\end{tabular}

Table 4 Number of the unknowns when $m \neq n$ and one is even the other is odd (Only " 1 " and " 2 " are used)

\begin{tabular}{ccccc}
\hline \multirow{\Sigma}{\Sigma}{} & SS-- & SA- & AS- & AA- \\
\hline $\boldsymbol{e}-\boldsymbol{o}$ & $\frac{m(n-1)}{4}$ & $\frac{m(n-1)}{4}$ & $\frac{(m-2)(n-1)}{4}$ & $\frac{(m-2)(n-1)}{4}$ \\
\hline $\boldsymbol{o - \boldsymbol { e }}$ & $\frac{(m-1) n}{4}$ & $\frac{(m-1)(n-2)}{4}$ & $\frac{(m-1) n}{4}$ & $\frac{(m-1)(n-2)}{4}$ \\
\hline
\end{tabular}

" 3 " and " 4 " symmetry planes can be only used if $m=n$.

Table 5 Number of the unknowns when $m=n$ and both are even or odd (Only " 3 " and " 4 " are used)

\begin{tabular}{lllll}
\hline \multirow{2}{s}{} & $-\mathbf{S S}$ & $-\mathbf{S A}$ & $-\mathbf{A S}$ & --AA \\
\hline e. & $\frac{m^{2}}{4}$ & $\frac{m^{2}}{4}-\frac{m}{2}$ & $\frac{m^{2}}{4}-\frac{m}{2}$ & $\left(\frac{m}{2}-1\right)^{2}$ \\
\hline o. & $\frac{m^{2}-1}{4}$ & $\left(\frac{m-1}{2}\right)^{2}$ & $\left(\frac{m-1}{2}\right)^{2}$ & $\frac{(m-3)(m-1)}{4}$ \\
\hline
\end{tabular}

All the symmetry planes can be only used if $m=n$.

Table 6 Number of the unknowns when $m=n$ and all the symmetry planes are used

\begin{tabular}{ccccc}
\hline \multirow{2}{*}{} & SSSS & SSAA & AASS & AAAA \\
\hline e. & $\frac{m^{2}}{4}-\sum_{i=0}^{\frac{m-2}{2}} i$ & $\frac{m(m-2)}{4}-\sum_{i=1}^{\frac{m-2}{2}} i$ & $\frac{m(m-2)}{4}-\sum_{i=1}^{\frac{m-2}{2}} i$ & $\frac{m(m-4)}{4}-\sum_{i=2}^{\frac{m-2}{2}} i$ \\
\hline o. & $\frac{(m-1)^{2}}{4}-\sum_{i=0}^{\frac{m-3}{2}} i$ & $\frac{(m-3)(m-1)}{4}-\sum_{i=1}^{\frac{m-3}{2}} i$ & $\frac{(m-1)^{2}}{4}-\sum_{i=0}^{\frac{m-3}{2}} i$ & $\frac{(m-3)(m-1)}{4}-\sum_{i=1}^{\frac{m-3}{2}} i$ \\
\hline
\end{tabular}

The tables give the relationship between the number of the shells and unknowns belong to different orth. groups. In the 
" $m \mathrm{x} n$ " column " $e$." means $m$ and $n$ are even. The "o." means both are odd and " $e-o$ " $m$ is even, $n$ is odd.

\section{Compatibility matrixes of the shell structures}

The structure of the compatibility matrix is based on the orth. system (groups). All the orth. groups create a symmetric sub-matrix. From these sub-matrixes the comp. matrix can be easily written in (3) and (4).

$$
\begin{aligned}
& \mathrm{D}_{12}=\left[\begin{array}{cccc}
{\left[\mathrm{M}_{S S}\right]} & \cdot & \cdot & 0 \\
\cdot & {\left[\mathrm{M}_{S A}\right]} & & \cdot \\
\cdot & & {\left[\mathrm{M}_{A S}\right]} & \cdot \\
0 & \cdot & \cdot & {\left[\mathrm{M}_{A A}\right]}
\end{array}\right] \\
& \mathrm{D}_{1234}=\left[\begin{array}{cccccc}
{\left[\mathrm{M}_{4 S}\right]} & \cdot & \cdot & \cdot & \cdot & 0 \\
\cdot & {\left[\mathrm{M}_{S S A A}\right]} & & & & \cdot \\
\cdot & & {\left[\mathrm{M}_{A A S S}\right]} & & & \cdot \\
\cdot & & & {\left[\mathrm{M}_{4 A}\right]} & & \cdot \\
\cdot & & & & {\left[\mathrm{M}_{S A}\right]} & \cdot \\
0 & . & . & . & \cdot & {\left[\mathrm{M}_{A S}\right]}
\end{array}\right]
\end{aligned}
$$

The sub-matrixes are needed to determine the comp. matrixes. All these matrixes are symmetric, their rows and columns number are equal with unknowns in the orth. groups. Let see an example; a 6x6 shell structure SS-- orth. group contains 9 unknowns, thus the size of the $\mathbf{M}_{\mathrm{SS}}$ matrix is $9 \times 9$ and etc.

In the following the unknowns are examined in the second quadrant, because the other unknowns are mapped from here by the help of antimetry and symmetry.

Using the properties of the symmetry and antimetry, 8 submatrixes $\left(\mathbf{M}_{\mathrm{SS}}, \mathbf{M}_{\mathrm{SA}}, \mathbf{M}_{\mathrm{AS}}, \mathbf{M}_{\mathrm{AA}}, \mathbf{M}_{4 \mathrm{~S}}, \mathbf{M}_{2 \mathrm{~S} 2 \mathrm{~A}}, \mathbf{M}_{2 \mathrm{~A} 2 \mathrm{~S}}\right.$, and $\left.\mathbf{M}_{4 \mathrm{~A}}\right)$ are determined in general closed form. The coefficient of the comp. matrixes is denoted with $\delta_{p q}$. To identify the unknowns in the quadrant set-theory is applied.The introduced method is applicable when " 1 " and " 2 " planes are used, because it is general in symmetric shell structure (see Table 2).

The different unknowns in the quadrant are denoted by $x$. Consider $x$ an element of the $U N$ set. With this set, unknowns can be described even and odd cases in different orth. groups as well. Indices $E$ means even, $O$ means odd case. The unknown does not exist (take into consideration) when it is intersected by an antimetry plane. In the subscripts the short of the different orth. groups are written.

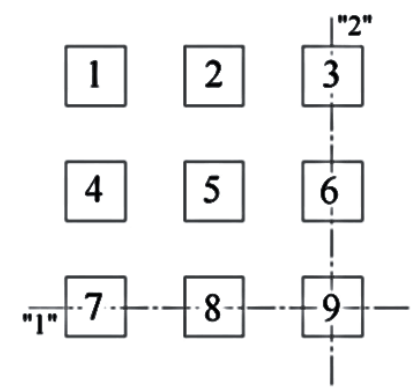

\section{fere} ferent orth. groups, but for the unknown identification only
the symmetry requirement is used (SS--, --SS and SSSS). The numbering can be seen on Fig. 9 and Fig. 10.

$$
U N=\left\{x \mid x \in N^{+}, x \leq \frac{m n}{4}\right\}
$$

$$
\begin{gathered}
I_{S S}^{E}=\left\{i \mid i \in N^{+}, i \leq \frac{m}{2}\right\} \\
J_{S S}^{E}=\left\{j \mid j \in N^{+}, j \leq \frac{n}{2}\right\}
\end{gathered}
$$

$$
\begin{gathered}
S_{1}^{E}=\left\{x \mid x \in U N, x=\left(\frac{m}{2}-1\right)\left(\frac{n}{2}\right)+j, j \in J\right\} \\
S_{2}^{E}=\left\{x \mid x \in U N, x=i \frac{n}{2}, i \in I\right\}
\end{gathered}
$$$$
\delta_{p q}=\left\{\begin{array}{l}
4 \delta_{11}, x \notin\left(S_{1} \cup S_{2}\right) \\
2 \delta_{11}, x \in\left(S_{1} \cup S_{2}\right) \backslash\left(S_{1} \cap S_{2}\right) \\
\delta_{11}, x \in\left(S_{1} \cap S_{2}\right) \\
p=q
\end{array}\right.
$$$$
\delta_{p q}=\left\{\begin{array}{l}
4 \delta_{12}, x=U N^{2} \backslash\left(S_{1} \cup S_{2}\right)^{2} \\
2 \delta_{12}, x=\left(S_{1} \cup S_{2}\right)^{2} \\
p \neq q
\end{array}\right.
$$

Let see an example; a 6x6 shell structure.

The SS orth. groups;

$$
\begin{gathered}
U N=\left\{x \mid x \in N^{+}, x \leq \frac{m n}{4}\right\}=\{1,2, \ldots 9\} \\
I_{S S}^{E}=\left\{i \mid i \in N^{+}, i \leq \frac{m}{2}\right\}=\{1,2,3\} \\
J_{S S}^{E}=\left\{j \mid j \in N^{+}, j \leq \frac{n}{2}\right\}=\{1,2,3\} \\
S_{1}^{E}=\left\{x \mid x \in U N, x=\left(\frac{m}{2}-1\right)\left(\frac{n}{2}\right)+j, j \in J\right\}=\{7,8,9\}
\end{gathered}
$$

Fig. 9 Location of the unknowns when $m$ and $n$ are even (6x6) 
$S_{2}^{E}=\left\{x \mid x \in U N, x=i \frac{n}{2}, i \in I\right\}=\{3,6,9\}$

(16)

$$
\delta_{p q}=\left\{\begin{array}{l}
4 \delta_{11}, x=\{1,2,4,5\} \\
2 \delta_{11}, x=\{3,6,7,8,\} \\
\delta_{11}, x=\{9\} \\
p=q
\end{array}\right.
$$

$$
\delta_{p q}=\left\{\begin{array}{l}
4 \delta_{12}, x=\left\{\begin{array}{l}
1-2,1-4,2-3,2-5, \\
4-5,4-7,5-6,5-8,
\end{array}\right\} \\
2 \delta_{12}, x=\{3-6,9-9,7-8,8-9,\} \\
p \neq q
\end{array}\right.
$$

The SA orth. groups;

$$
\begin{gathered}
I_{S A}^{E}=\left\{i \mid i \in N^{+}, i \leq \frac{m}{2}\right\}=\{1,2,3\} \\
J_{S A}^{E}=\left\{j \mid j \in N^{+}, j \leq \frac{n-2}{2}\right\}=\{1,2\} \\
S_{1}^{E}=\left\{x \mid x \in U N, x=\left(\frac{m}{2}-1\right)\left(\frac{n}{2}\right)+j, j \in J\right\}=\{7,8\} \\
A_{2}^{E}=\left\{x \mid x \in U N, x=i \frac{n}{2}, i \in I\right\}=\{3,6,9\} \\
\delta_{p q}=\left\{\begin{array}{l}
4 \delta_{11}, x=\{1,2,4,5\} \\
2 \delta_{11}, x=\{7,8\} \\
\delta_{11}, x=\{\varnothing\} \\
p=q
\end{array}\right. \\
\delta_{p q}=\left\{\begin{array}{l}
4 \delta_{12}, x=\left\{\begin{array}{l}
1-2,1-4,2-5, \\
4-5,4-7,5-8,
\end{array}\right\} \\
2 \delta_{12}, x=\{7-8\}
\end{array}\right]
\end{gathered}
$$

The AS groups

$$
\begin{aligned}
& I_{A S}^{E}=\left\{i \mid i \in N^{+}, i \leq \frac{m-2}{2}\right\}=\{1,2\} \\
& J_{A S}^{E}=\left\{j \mid j \in N^{+}, j \leq \frac{n}{2}\right\}=\{1,2,3\}
\end{aligned}
$$

$$
A_{1}^{E}=\left\{x \mid x \in U N, x=\left(\frac{m}{2}-1\right)\left(\frac{n}{2}\right)+j, j \in J\right\}=\{7,8,9\}
$$

$$
S_{2}^{E}=\left\{x \mid x \in U N, x=i \frac{n}{2}, i \in I\right\}=\{3,6\}
$$

$$
\delta_{p q}=\left\{\begin{array}{l}
4 \delta_{11}, x=\{1,2,4,5\} \\
2 \delta_{11}, x=\{3,6\} \\
\delta_{11}, x=\{\varnothing\} \\
p=q
\end{array}\right.
$$

$$
\delta_{p q}=\left\{\begin{array}{l}
4 \delta_{12}, x=\left\{\begin{array}{l}
1-2,1-4,2-3, \\
2-5,4-5,5-6,
\end{array}\right\} \\
2 \delta_{12}, x=\{3-6\} \\
p \neq q
\end{array}\right.
$$

The AA groups

$$
\begin{gathered}
I_{A A}^{E}=\left\{i \mid i \in N^{+}, i \leq \frac{m-2}{2}\right\}=\{1,2\} \\
J_{A A}^{E}=\left\{j \mid j \in N^{+}, j \leq \frac{n-2}{2}\right\}=\{1,2\} \\
A_{1}^{E}=\left\{x \mid x \in U N, x=\left(\frac{m}{2}-1\right)\left(\frac{n}{2}\right)+j, j \in J\right\}=\{7,8\} \\
A_{2}^{E}=\left\{x \mid x \in U N, x=i \frac{n}{2}, i \in I\right\}=\{3,6\} \\
\delta_{p q}=\left\{\begin{array}{l}
4 \delta_{11}, x=\{1,2,4,5\} \\
2 \delta_{11}, x=\{\varnothing\} \\
\delta_{11}, x=\{\varnothing\} \\
p=q
\end{array}\right. \\
\delta_{p q}=\left\{\begin{array}{l}
4 \delta_{12}, x=\left\{\begin{array}{l}
1-2,1-4, \\
2-5,4-5,
\end{array}\right\} \\
2 \delta_{12}, x=\{\varnothing\} \\
p \neq q
\end{array}\right.
\end{gathered}
$$

Another example $6 \times 5$ shell structure

The SS and SA orth. groups;

$$
\begin{aligned}
& U N=\left\{x \mid x \in N^{+}, x \leq \frac{m(n-1)}{4}\right\}=\{1,2, \ldots 6\} \\
& I_{S S}^{E O}=I_{S A}^{E O}=\left\{i \mid i \in N^{+}, i \leq \frac{m}{2}\right\}=\{1,2,3\}
\end{aligned}
$$

$$
J_{S S}^{E O}=J_{S A}^{E O}=\left\{j \mid j \in N^{+}, j \leq \frac{n-1}{2}\right\}=\{1,2\}
$$

$S_{1}^{E}=\left\{x \mid x \in U N, x=\left(\frac{m}{2}-1\right)\left(\frac{n}{2}-1\right)+j, j \in J\right\}=\{5,6\}$

$$
S_{2}^{E O}=A_{2}^{E O}=\varnothing
$$

$$
\begin{gathered}
\delta_{p q}=\left\{\begin{array}{l}
4 \delta_{11}, x=\{1,2,3,4\} \\
2 \delta_{11}, x=\{5,6\} \\
\delta_{11}, x=\{\varnothing\} \\
p=q
\end{array}\right. \\
\delta_{p q}=\left\{\begin{array}{l}
4 \delta_{12}, x=\left\{\begin{array}{l}
1-2,1-3,2-4, \\
3-4,3-5,4-6, \\
2 \delta_{12}, x=\{5-6\} \\
p \neq q
\end{array}\right.
\end{array}\right.
\end{gathered}
$$


The AS and AA orth. groups;

$$
\begin{gathered}
I_{A S}^{E O}=I_{A A}^{E O}=\left\{i \mid i \in N^{+}, i \leq \frac{m-2}{2}\right\}=\{1,2\} \\
J_{A S}^{E O}=J_{A A}^{E O}=\left\{j \mid j \in N^{+}, j \leq \frac{n-1}{2}\right\}=\{1,2\} \\
A_{1}^{E}=\left\{x \mid x \in U N, x=\left(\frac{m}{2}-1\right)\left(\frac{n}{2}-1\right)+j, j \in J\right\}=\{5,6\} \\
S_{2}^{E O}=A_{2}^{E O}=\varnothing \\
\delta_{p q}=\left\{\begin{array}{l}
4 \delta_{11}, x=\{1,2,3,4\} \\
2 \delta_{11}, x=\{\varnothing\} \\
\delta_{11}, x=\{\varnothing\} \\
p=q
\end{array}\right. \\
2 \delta_{12}, x=\{\varnothing\} \\
p \neq q
\end{gathered}
$$

If all the symmetry planes are used ("1", "2", "3" and "4") " 2 " and " 3 " symmetry planes determine the unknowns (Fig. 11 and 12) In case of the $m$ is even the unknowns which are located " 2 " and " 3 " planes must be symmetric. In case of the $m$ is odd the unknown which are located " 3 " must be symmetric.

The different unknowns in the third eighth of space are denoted by $x$. Consider $x$ an element of the $U N$ set. With this set, unknowns can be described even and odd cases in different orth. groups as well. Indices $E$ means even, $O$ means odd case. The unknown does not exist when it is intersected by an antimetry plane.

Let see two examples. If $m=6$ the number of the unknowns can be seen on Fig. 11.

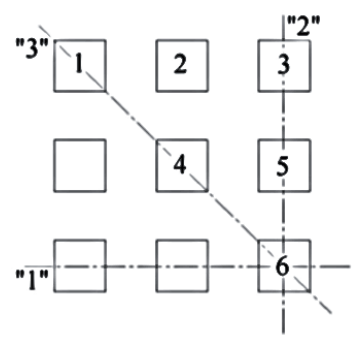

Fig. 11 Location of the unknowns when $m$ is even and all the symmetry planes are used $(6 \times 6)$

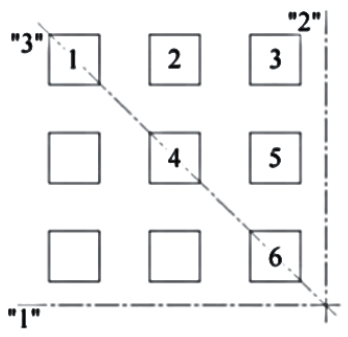

Fig. 12 Location of the unknowns when $m$ is odd and all the symmetry planes are used $(7 \times 7)$

$$
U N^{E}=\left\{x \mid x \in N^{+}, x \leq \frac{m^{2}}{4}-\sum_{i=0}^{\frac{m-2}{2}} i\right\}=\{1,2, \ldots 6\}
$$

The unknowns which are located on the symmetry /antimetry planes can be calculated:

$$
I^{E}=\left\{i \mid i \in N, i \leq \frac{m-2}{2}\right\}=\{0,1, \ldots 2\}
$$

$$
K^{E}=\left\{k \mid k \in N, k \leq \frac{m-2}{2}-i\right\}=\{2,1 \ldots 0\}
$$

The 4S group:

$$
\begin{gathered}
S_{2}^{E}=\left\{x \mid x \in U N, x=\sum_{0}^{i} \frac{m}{2}-i, i \in I\right\}=\{3,5,6\} \\
S_{3}^{E}=\left\{x \mid x \in U N, x=\sum_{0}^{i} \frac{m}{2}-i-k, i \in I, k \in K\right\}=\{1,4,6\} \\
\delta_{p q}=\left\{\begin{array}{l}
8 \delta_{11}, x \notin\left(S_{2} \cup S_{3}\right)=\{2\} \\
4 \delta_{11}, x \in\left(S_{2} \cup S_{3}\right) \backslash\left(S_{2} \cap S_{3}\right)=\{1,3,4,5\} \\
\delta_{11}, x \in\left(S_{2} \cap S_{3}\right)=\{6\} \\
p=q
\end{array}\right. \\
\delta_{p q}=\left\{\begin{array}{l}
8 \delta_{12}, x=\left(U N^{2} \backslash S_{2}^{2}\right)\{1-2,2-3,2-4,4-5\} \\
4 \delta_{12}, x=\left(S_{2}^{2}\right)=\{3-5,5-6\} \\
p \neq q
\end{array}\right.
\end{gathered}
$$

The 2S2A group:

$$
I^{E}=\left\{i \mid i \in N, i \leq \frac{m}{2}-2\right\}=\{0,1\}
$$

$$
K^{E}=\left\{k \mid k \in N, k \leq \frac{m}{2}-1-i\right\}=\{1,0\}
$$

$$
\begin{gathered}
S_{2}^{E}=\left\{x \mid x \in U N, x=\sum_{0}^{i} \frac{m}{2}-i, i \in I\right\}=\{3,5\} \\
A_{3}^{E}=\{1,4,6\}
\end{gathered}
$$

$$
\delta_{p q}=\left\{\begin{array}{l}
8 \delta_{11}, x \notin\left(S_{2} \cup A_{3}\right)=\{2\} \\
4 \delta_{11}, x \in\left(S_{2} \cup A_{3}\right) \backslash\left(S_{2} \cap A_{3}\right)=\{3,5\} \\
\delta_{11}, x \in\left(S_{2} \cap A_{3}\right)=\varnothing \\
p=q
\end{array}\right.
$$

$$
\delta_{p q}=\left\{\begin{array}{l}
8 \delta_{12}, x=\left(U N^{2} \backslash S_{2}^{2}\right)=\{2-3\} \\
4 \delta_{12}, x=\left(S_{2}^{2}\right)=\{3-5\} \\
p \neq q
\end{array}\right.
$$

The 2A2S group:

$$
I^{E}=\left\{i \mid i \in N, i \leq \frac{m}{2}-2\right\}=\{0,1\}
$$




$$
\begin{gathered}
K^{E}=\left\{k \mid k \in N, k \leq \frac{m}{2}-1-i\right\}=\{1,0\} \\
A_{2}^{E}=\{3,5,6\} \\
S_{3}^{E}=\left\{x \mid x \in U N, x=\sum_{0}^{i} \frac{m}{2}-i-k, i \in I, k \in K\right\}=\{1,4\} \\
\delta_{p q}=\left\{\begin{array}{l}
8 \delta_{11}, x \notin\left(A_{2} \cup S_{3}\right)=\{2\} \\
4 \delta_{11}, x \in\left(A_{2} \cup S_{3}\right) \backslash\left(A_{2} \cap S_{3}\right)=\{1,4\} \\
\delta_{11}, x \in\left(A_{2} \cap S_{3}\right)=\varnothing \\
p=q
\end{array}\right. \\
\delta_{p q}=\left\{\begin{array}{l}
8 \delta_{12}, x=\left(U N^{2} \backslash S_{2}^{2}\right)=\{1-2,2-4\} \\
4 \delta_{12}, x=\left(S_{2}^{2}\right)=\varnothing \\
p \neq q
\end{array}\right.
\end{gathered}
$$

The 4A group:

$$
\begin{gathered}
A_{2}^{E}=\left\{x \mid x \in U N, x=\sum_{0}^{i} \frac{m}{2}-i, i \in I\right\}=\{3,5,6\} \\
A_{3}^{E}=\left\{x \mid x \in U N, x=\sum_{0}^{i} \frac{m}{2}-i-k, i \in I, k \in K\right\}=\{1,4,6\} \\
\delta_{p q}=\left\{\begin{array}{l}
8 \delta_{11}, x \notin\left(A_{2} \cup A_{3}\right)=\{2\} \\
4 \delta_{11}, x \in\left(A_{2} \cup A_{3}\right) \backslash\left(A_{2} \cap A_{3}\right)=\varnothing \\
\delta_{11}, x \in\left(A_{2} \cap A_{3}\right)=\varnothing \\
p=q
\end{array}\right. \\
\delta_{p q}=\left\{\begin{array}{l}
8 \delta_{12}, x=\left(U N^{2} \backslash S_{2}^{2}\right)=\varnothing \\
4 \delta_{12}, x=\left(S_{2}^{2}\right)=\varnothing \\
p \neq q
\end{array}\right.
\end{gathered}
$$

If $m$ is odd and all symmetry planes are used the process of the unknown calculation modified a bit (Fig. 12). The $S_{2}$ set is empty, because there is no unknown which is intersected with this plane. The $\mathrm{S}_{3}$ is stayed unchanging.

If $m=7$, the number of the unknowns

$$
\begin{gathered}
U N^{O}=\left\{x \mid x \in N^{+}, x \leq \frac{(m-1)}{4} \sum_{i=0}^{\frac{m-3}{2}} i\right\}=\{1,2, \ldots 6\} \\
I^{O}=\left\{i \mid i \in N, i \leq \frac{(m-1)}{2}-1\right\}=\{0,1 \ldots 2\} \\
K^{O}=\left\{k \mid k \in N, k \leq \frac{m-1}{2}-1-i\right\}=\{2,1, \ldots 0\} \\
S_{3}^{E}=\left\{x \mid x \in U N, x=\sum_{0}^{i} \frac{m-1}{2}-i-k, i \in I\right\}=\{1,4,6\}
\end{gathered}
$$

$$
\begin{gathered}
\delta_{p q}=\left\{\begin{array}{l}
8 \delta_{11}, x \notin\left(S_{2} \cup S_{3}\right)=\{2,3,5\} \\
4 \delta_{11}, x \in\left(S_{2} \cup S_{3}\right) \backslash\left(S_{2} \cap S_{3}\right)=\{1,4,6\} \\
\delta_{11}, x \in\left(S_{2} \cap S_{3}\right)=\varnothing \\
p=q
\end{array}\right. \\
\delta_{p q}=\left\{\begin{array}{l}
8 \delta_{12}, x=\left(U N^{2} \backslash S_{2}^{2}\right)=\left\{\begin{array}{l}
1-2,2-3,2-4, \\
3-5,4-5,5-6
\end{array}\right\} \\
4 \delta_{12}, x=\left(S_{2}^{2}\right)=\varnothing \\
p \neq q
\end{array}\right.
\end{gathered}
$$

\section{Conclusion}

Stiffened symmetric shell structure analysis is introduced in different symmetry cases. The number of the shell fields determines the process of the computational work. The comp. matrix of the structure can be easily created from the sub-matrixes. These sub-matrixes are determined by the help of the orth. systems. There are up to three different orth. systems can be applicable, depending on the applied symmetry planes and number of the shell fields. The "1" and "2" symmetry planes can be used, if $m=n$ or $m \neq n$. This orth. system creates 4 orth. groups. The " 3 " and " 4 " symmetry planes can be used, if $m=n$. This orth. system contains of 4 orth. groups as well. Application of higher order symmetry (all the symmetry planes are used) make the easier computational work possible, but here is needed to complete with lower orth. groups. This orth. system contains 4 higher and 2 lower orth. groups.It is clearly seen usage of higher order of symmetry reduce the number of the unknowns in one equation thus the computational work, but means a stricter requirement for outer load at the same time.

\section{References}

Adeleke, S. A. (1983) On symmetry of shells. Journal of Elasticity. 13 (2). pp. 111-119. DOI: $10.1007 /$ bf00041228

Adeleke, S. A. (1985) On possible symmetries of shells. Archive for Rational Mechanics and Analysis. 88 (3). pp. 195-207. DOI: $10.1007 / \mathrm{bf00752110}$

Eremeyev, V. A., Pietraszkiewicz, W. (2006) Local Symmetry Group in the General Theory of Elastic Shells. Journal of Elasticity. 85. pp. 125-152. DOI: $10.1007 / \mathrm{s} 10659-006-9075-\mathrm{z}$

Lucas, A. H. (1959) The stress Analysis of Railway Coach by the Argyris Matrix Force Method of Structural Analysis. Burgess Hill. Norris Brothers report No. 59 .

Lucas, A. H. (1960) The Stress Analysis of Railway Coaches. Engineering. 189. pp. 669-671.

Lucas, A. H. (1963) Structural analysis of an integral van trailer. Automotive Design Engineering. 2 (8). pp. 38-42.

Tytyunnikov, N. P., Shklyarchuk, F. N. (2008) Analysis of a strengthened weakly conical shell using a complete system of orthogonal functions on an arbitrary contour of its transverse cross-section. Mechanics of Solids. 43 (4). pp. 623-634. DOI: $10.3103 / \mathrm{s} 0025654408040109$ 